\title{
Distances and Evolutionary States of Supernova Remnant G18.9-1.1 and Candidate G28.6+0.0
}

\author{
Sujith Ranasinghe ${ }^{1 *}$, Denis Leahy ${ }^{1}$, Wenwu Tian ${ }^{1,2}$ \\ ${ }^{1}$ Department of Physics \& Astronomy, University of Calgary, Calgary, Canada \\ ${ }^{2}$ National Astronomical Observatories, CAS, Beijing, China \\ Email: *syranasi@ucalgary.ca
}

How to cite this paper: Ranasinghe, S., Leahy, D. and Tian, W.W. (2020) Distances and Evolutionary States of Supernova Remnant G18.9-1.1 and Candidate G28.6+0.0. Journal of High Energy Physics, Gravitation and Cosmology, 6, 9-19.

https://doi.org/10.4236/jhepgc.2020.61002

Received: October 11, 2019

Accepted: November 16, 2019

Published: November 19, 2019

Copyright $\odot 2020$ by author(s) and Scientific Research Publishing Inc. This work is licensed under the Creative Commons Attribution International License (CC BY 4.0).

http://creativecommons.org/licenses/by/4.0/

\begin{abstract}
HI spectra of the supernova remnant G18.9-1.1 and the supernova remnant candidate G28.6+0.0 are analyzed. We compared the spectra to ${ }^{13} \mathrm{CO}$ emission spectra and to spectra of HII regions in the area to determine kinematic distances. G18.9-1.1 is at $2.1 \pm 0.4 \mathrm{kpc}$ and G28.6+0.0 is at $9.0 \pm 0.3 \mathrm{kpc}$ from the Sun. Using the published X-ray spectra of G18.9-1.1, we apply supernova remnant models for shocked-ISM temperature and emission measure. We find that G18.9-1.1 has low, but not atypical, explosion energy of $\approx 3 \times 10^{50}$ erg and is in a low-density region of the ISM, $\sim 0.1 \mathrm{~cm}^{-3}$. It has age $\sim 3700 \mathrm{yr}$ if the ejecta mass is $1.4 \mathrm{M}_{\odot}$, typical of Type Ia SNe, or $\sim 4700 \mathrm{yr}$ if the ejecta mass is $5 \mathrm{M}_{\odot}$ typical of core-collapse $\mathrm{SN}$. The candidate G28.6+0.0 does not have reported X-ray emission, so we apply a basic Sedov model. The Sedov age is $\sim 600 \mathrm{yr}$ if the ISM density is $1 \mathrm{~cm}^{-3}$ but could be as old as $\sim 6000 \mathrm{yr}$ if the ISM density is as high as $100 \mathrm{~cm}^{-3}$.
\end{abstract}

\section{Keywords}

Supernova Remnants, Radio Continuum, Radio Lines

\section{Introduction}

Supernova remnants (SNRs) remain an important area of study due to their role in interstellar medium (ISM) and Galactic evolution. To determine the basic parameters (e.g. age and size) of a SNR, a distance estimation is necessary. There are 294 Galactic SNRs [1], and 262 SNRs in the area covered by the VLA Galactic Plane Survey (VGPS). Distances to 28 of the radio-bright SNRs ${ }^{1}$ were determined by previous work (see [2] [3] [4] and [5]). Two more distances to SNRs were estimated by [6], using data from the $\mathrm{HI}, \mathrm{OH}$, Recombination line survey ${ }^{1}$ Two are no longer classified as SNRs ([1] [12]). 
of the Milky Way (THOR). Furthermore, the evolutionary state of 15 SNRs was determined by [7].

For this work, we chose radio-faint SNRs that were omitted from the previous selection, by carefully investigating their HI channel maps. Due to its diffuse nature, determining the distance to the SNR G18.9-1.1 using HI absorption spectra has been a difficult task. First detected by [8], the morphology, spectrum and presence of the center bar directed it to be classified as a composite SNR ([9] [10]). Reference [11] determined a radio spectral index of $-0.39 \pm 0.03\left(S_{v} \propto v^{\alpha}\right)$ for the SNR.

The SNR candidate G28.6+0.0 (G28.56+0.00 in [12]) is located in the G28.6-0.1 complex which consists of HII regions and radio sources. Initially [13] suggested a shell-like object to be an SNR due to its non-thermal radio spectrum. However, [14] presented an X-ray analysis of the SNR and found that it emits predominantly synchrotron X-rays from the shell and retained the original SNR ID. Reference [13] argued that the integrated flux density at $20 \mathrm{~cm}$ for G28.6+0.0 was clearly higher than the $6 \mathrm{~cm}$ and $3 \mathrm{~cm}$ flux densities to suggest the non-thermal nature of the SNR candidate. Using THOR data and the $1400 \mathrm{MHz}$ radio continuum data from the VGPS data along with the Spitzer GLIMPSE $8.0 \mu \mathrm{m}$ and Spitzer MIPSGAL $24 \mu \mathrm{m}$ data, [12] classified the object as a SNR candidate.

In this paper, we present the $\mathrm{HI}$ and ${ }^{13} \mathrm{CO}$ data and analysis in Section 2 . We derive distances for the SNR and SNR candidate in Section 3. Evolutionary models for the two objects are presented in Section 4.

\section{Data and Analysis}

The basic parameters of the SNR G18.9-1.1 and the SNR candidate G28.6-0.0, were obtained from the catalogue of Galactic SNRs [1] and the catalogue of High Energy Observations of Galactic Supernova Remnants (http://www.physics.umanitoba.ca/snr/SNRcat/ [15]).

\section{Radio and ${ }^{13}$ CO Emission Data}

The $1420 \mathrm{MHz}$ and $\mathrm{HI}$ data were retrieved from the [16] and the ${ }^{13} \mathrm{CO}$ spectral line from the Galactic Ring Survey of the Five College Radio Astronomical Observatory (FCRAO) $14 \mathrm{~m}$ telescope [17]. We followed the method of HI absorption spectra construction, spectral and error analysis as outlined by [18] and [2].

We used MEANLEV, a software program in the DRAO EXPORT package to construct $\mathrm{HI}$ absorption spectra. A main advantage of MEANLEV is that, the "on" (source) and "off" (background) spectra could be extracted by user-specified threshold continuum brightness $\left(T_{\mathrm{B}}\right)$ levels. Once a spatial region, normally a box is selected, the source and background spectra are extracted using pixels above (for source) and below (for background) the given $T_{\mathrm{B}}$ level. This maximizes the contrast difference in $\left(T_{\mathrm{B}}\right)$, which maximizes the signal-to-noise in the HI spectrum.

The THOR survey covers an area between the Galactic longitudes $14.5^{\circ}$ and 
$67.4^{\circ}$ and latitudes $-1.25^{\circ}$ and $1.25^{\circ}$ with a continuum angular resolution of $25^{\prime \prime}$ and HI data angular resolution of $40 "$ and spectral resolution of $1.6 \mathrm{~km} \cdot \mathrm{s}^{-1}$ [19]. We constructed HI emission spectra using the THOR data for the same source (on) and background (off) regions as chosen for the VGPS spectra. This was done in order to assess whether we could obtain an improvement of the spectra. However, we found there to be almost no difference between the THOR and the VGPS spectra: the prominent emission and absorption features of the source regions were present in the spectra from both surveys. Even though the THOR data has a higher angular resolution, the spectral resolution is comparable to the VGPS data. Thus, for this analysis, we found that the VGPS data was sufficient.

To estimate the kinematic distances to SNRs, a reliable rotation curve is necessary. For this work, we adopt the universal rotation curve (URC) of [20] with the [21] parameters, where the Galactocentric radius of $R_{0}=8.34 \pm 0.16 \mathrm{kpc}$ and orbital velocity of the sun $V_{0}=241 \pm 8 \mathrm{~km} \cdot \mathrm{s}^{-1}$.

\section{Results}

\subsection{SNR G18.9-1.1}

The diffuse nature of the SNR G18.9-1.1 has made extracting absorption spectra quite difficult, and care needs to be taken to avoid being dominated by noise. An inspection of the HI channel maps by [10] found a depression at $18 \mathrm{~km} \cdot \mathrm{s}^{-1}$ giving a distance of $2 \mathrm{kpc}$ to the SNR. However, [10] doesn't present HI absorption spectra. Using red clump stars [22] estimated the distance to be $1.8 \pm 0.2 \mathrm{kpc}$, an estimation consistent with [10].

The SNR consists of two brighter arcs (A \& B in Figure 1). We extracted multiple spectra for both regions and found that arc B's brightest region produces the best absorption spectrum. Figure 2 shows the HI emission spectra of the source region and the background region (top panel). The bottom panel shows the HI absorption spectrum. The HI channel maps reveal that the absorption/emission features seen in the negative velocity range are likely dominated by noise. There is no clear evidence of absorption up to the tangent point.

The absorption features seen at $\sim 40$ and $\sim 95 \mathrm{~km} \cdot \mathrm{s}^{-1}$ are false features that have $\mathrm{HI}$ emission features coincidentally in the background region. The only real absorption feature is seen at $\sim 18-23 \mathrm{~km} \cdot \mathrm{s}^{-1}$. This feature appears consistently in both arcs A \& B. Figure 3 is the image of HI channel maps averaged between 18.32 and $23.27 \mathrm{~km} \cdot \mathrm{s}^{-1}$. This shows the absorption matching the continuum morphology of arcs $\mathrm{A}$ and $\mathrm{B}$, confirming that the absorption is real. We have used different combinations of the HI channel maps to determine the velocity range where the absorption occurs. Using the velocity $23 \mathrm{~km} \cdot \mathrm{s}^{-1}$ of the upper edge of the absorption feature, we find the SNR is at a distance of $2.1 \pm 0.4 \mathrm{kpc}$.

\subsection{SNR Candidate G28.6+0.0}

We present the $1420 \mathrm{MHz}$ radio continuum image of SNR candidate G28.6+0.0 including the HII regions and radio sources of the G28.6-0.1 complex in Figure 4. 
The labelling convention is the same as that in [13]. The HII region labelled as D by [13] is in fact two separate HII regions G028.610+0.020 and G028.600-0.011 with the radio recombination line (RRL) velocities of 96.6 and $41.9 / 92.8 \mathrm{~km} \cdot \mathrm{s}^{-1}$, respectively [23]. For this work, we re-label the two HII regions as D and D' (see

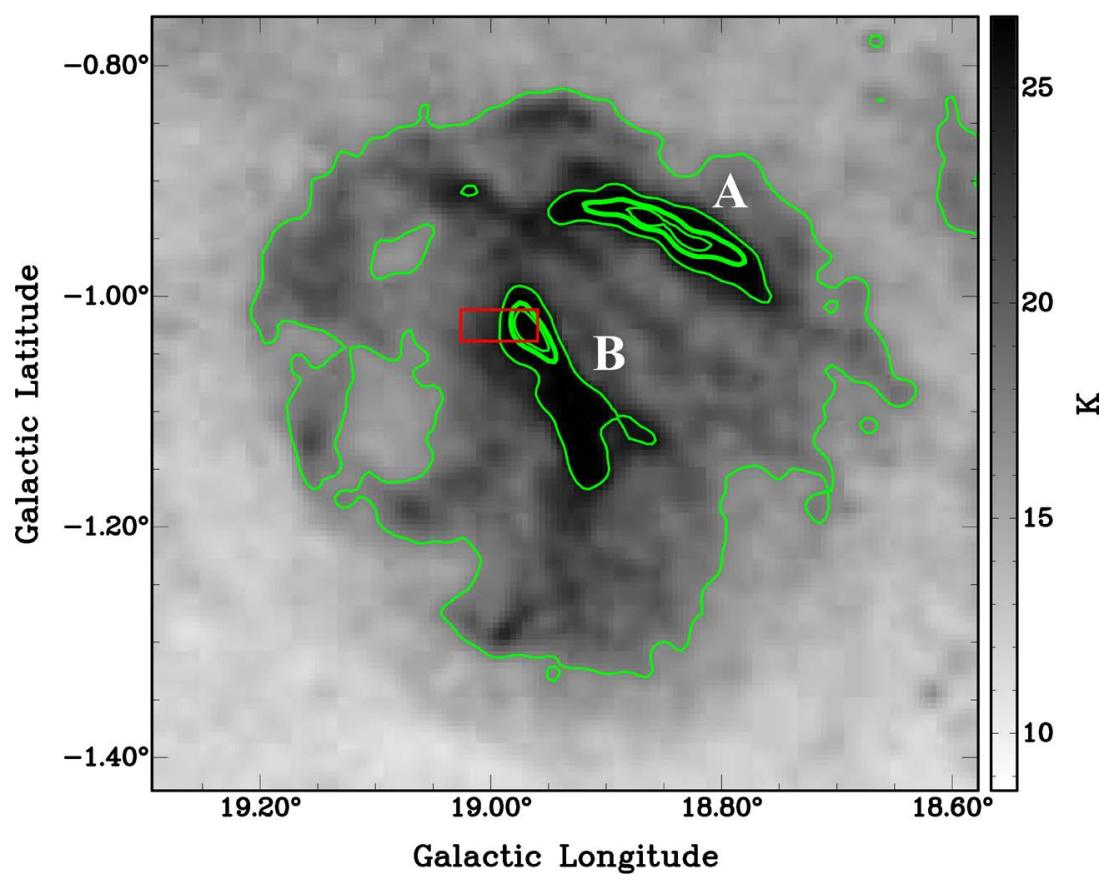

Figure 1. VGPS $1420 \mathrm{MHz}$ Continuum image of G18.9-1.1 with green contours at continuum brightness temperatures of 17.5, 25, 30 and $32 \mathrm{~K}$. The red box denotes the region used for the HI absorption spectrum extraction.

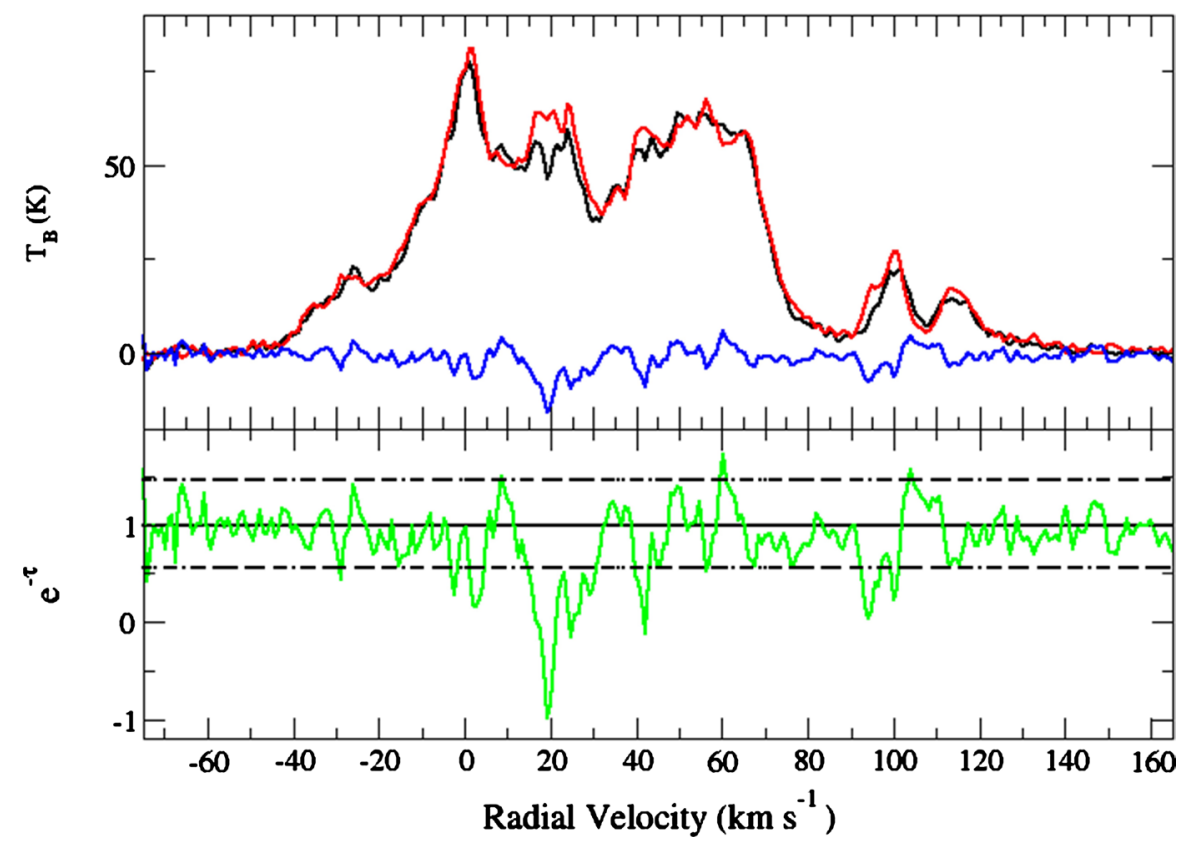

Figure 2. G18.9-1.1 absorption spectrum. Top panel: emission spectra for the source (black), background (red) and difference (blue). Bottom panel: absorption spectrum (green) and the $2 \sigma$ noise level (Dashed) of the HI absorption spectrum. 


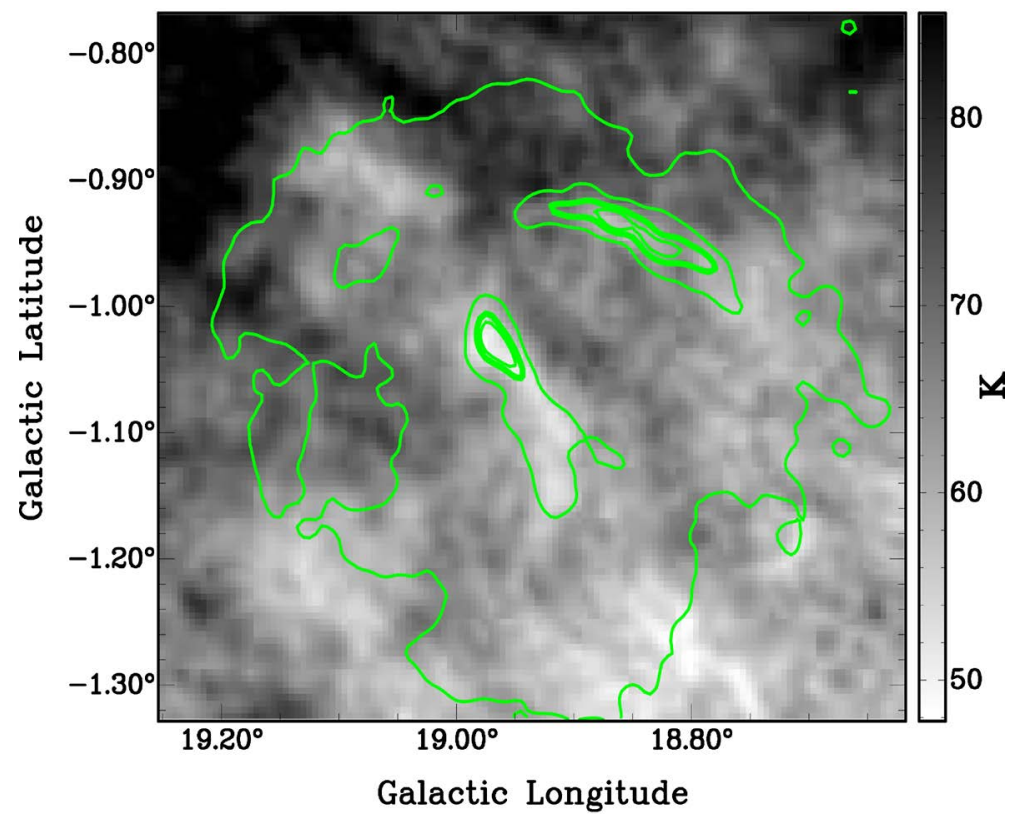

Figure 3. Combined HI channel maps +18.32 to $+23.27 \mathrm{~km} \cdot \mathrm{s}^{-1}$ for the SNR G18.9-1.1. $1420 \mathrm{MHz}$ continuum contour lines are in green at 17.5, 25, 30 \& $32 \mathrm{~K}$.

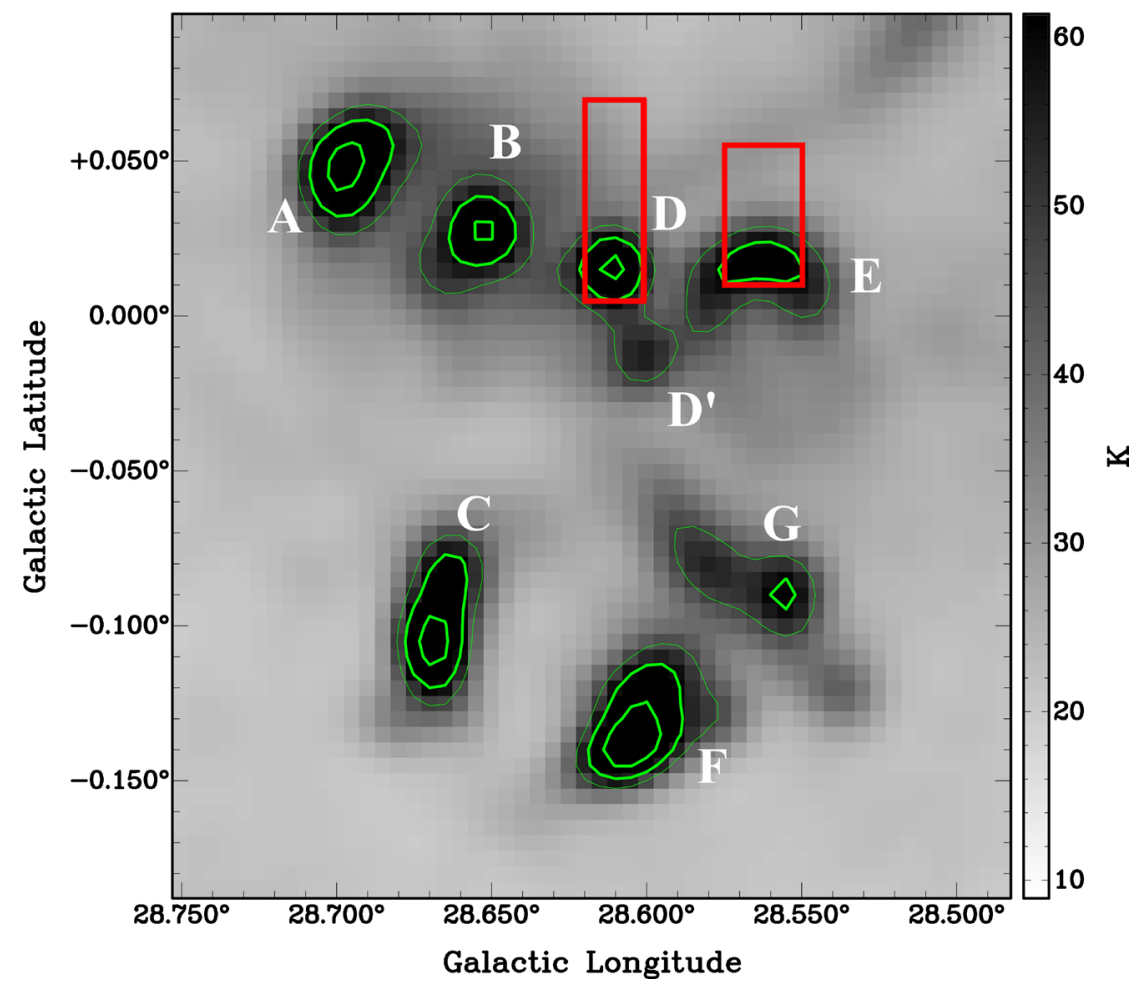

Figure 4. VGPS $1420 \mathrm{MHz}$ Continuum image of the G28.6-0.1 complex, with green contours at continuum brightness temperatures of 48,60 and $80 \mathrm{~K}$. Red boxes denote the regions used for the $\mathrm{HI}$ absorption spectrum extraction.

Figure 4). We extracted HI absorption spectra for the SNR candidate and HII region (D), which are shown in Figure 5. The scaled ${ }^{13} \mathrm{CO}$ emission spectra of the background (black curve) and source (purple curve) regions are shown in 
the bottom panels of Figure 5.

The HI absorption feature seen in the negative velocity range (near -40 $\mathrm{km} \cdot \mathrm{s}^{-1}$ ) is false, because HI emission is found coincidentally in the chosen background region (Figure 6). Furthermore, this feature is seen in both the SNR candidate and HII region spectra. For both sources, HI absorption is seen up to the tangent point, which yields a lower limit distance of $7.3 \mathrm{kpc}$ (the tangent point distance). ${ }^{13} \mathrm{CO}$ emission features are seen at the velocities $\sim 40, \sim 75$ and
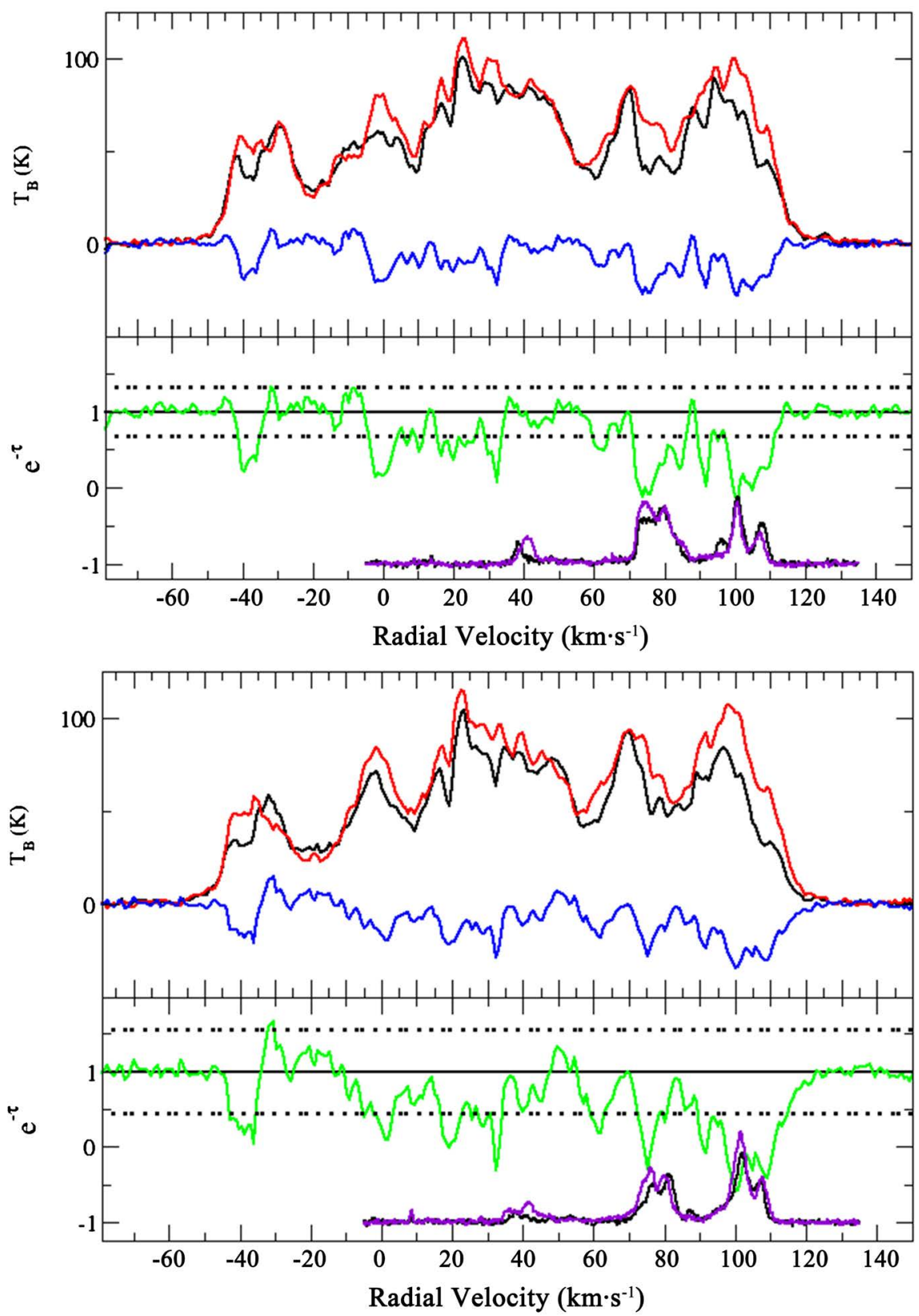

Figure 5. HI absorption spectra for the SNR candidate G28.6+0.0 (top) and the HII region G028.610+0.020 (bottom). For both panels, HI emission spectra for the source are in black, HI background spectra are red and the difference spectra are blue. The HI absorption spectra are green, their $2 \sigma$ noise levels are the dashed lines. The scaled ${ }^{13} \mathrm{CO}$ emission spectra of the background are in black and of the source are in purple. 
$\sim 100 \mathrm{~km} \cdot \mathrm{s}^{-1}$, and are consistent with the HI absorption features for both objects. Thus, the ${ }^{13} \mathrm{CO}$ clouds at these velocities are in front of the objects.

The absorption spectra of both the SNR candidate and HII region G028.610 +0.020 are nearly identical: the differences are less than the noise level (see Figure 5). This has been verified by examining HI channel maps. The HII region G028.610+0.020 has a RRL velocity of $96.6 \mathrm{~km} \cdot \mathrm{s}^{-1}$ and is at a distance of $9 \mathrm{kpc}(\mathrm{D}$ in Table 1). It is likely that the SNR candidate is related to the HII region because

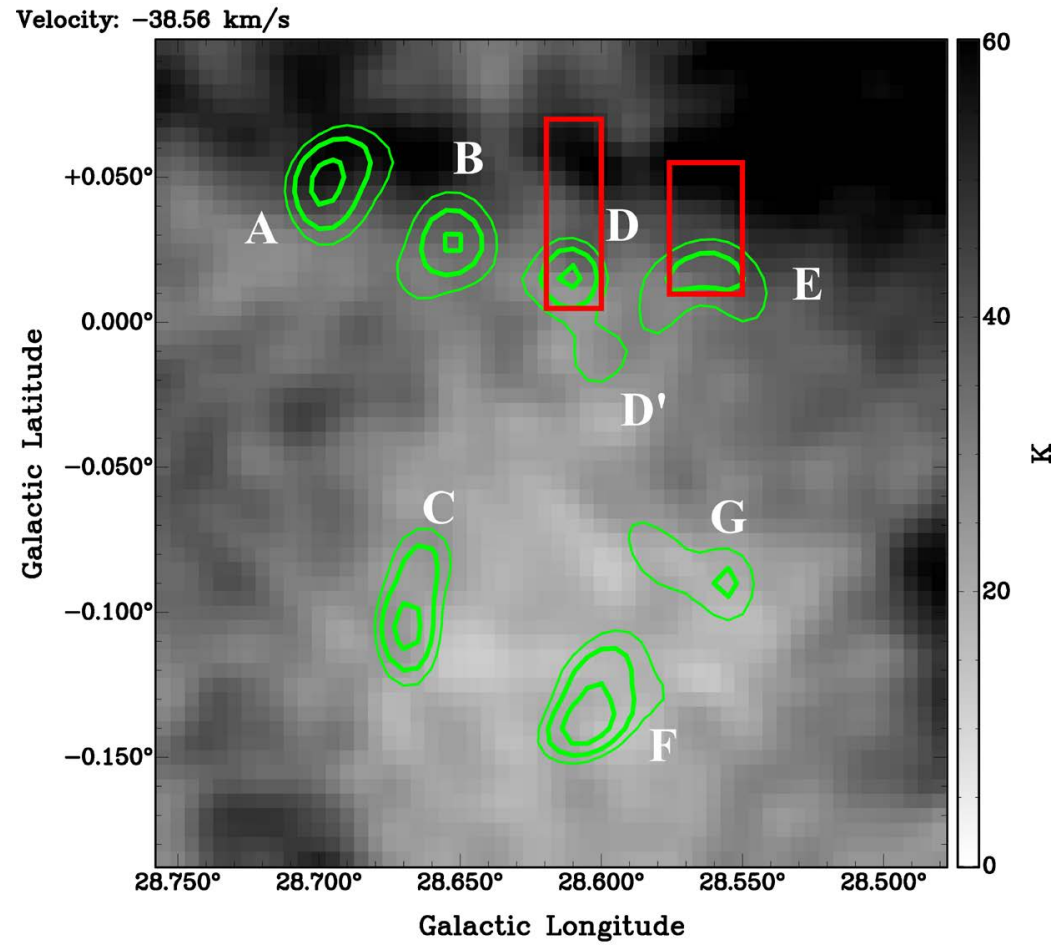

Figure 6. VGPS HI channel map of G28.6-0.1 complex at $-38.56 \mathrm{~km} \cdot \mathrm{s}^{-1}$. The dark shading in the upper parts of the red boxes indicates coincidental HI emission in the background region, which yields a false absorption feature. The green contours are at continuum brightness temperatures of 48,60 and $80 \mathrm{~K}$.

Table 1. Distances and radial velocities of the objects in G28.6-0.1 complex.

\begin{tabular}{ccccccc}
\hline Source & $\operatorname{VLSR}^{\mathrm{a}}\left(\mathrm{km} \cdot \mathrm{s}^{-1}\right)$ & $\mathrm{R}^{\mathrm{b}}(\mathrm{kpc})$ & $\mathrm{d}^{\mathrm{b}}(\mathrm{kpc})$ & $\operatorname{Refs}^{\mathrm{b}}$ & $\mathrm{R}^{\mathrm{c}}(\mathrm{kpc})$ & $\mathrm{d}^{\mathrm{c}}(\mathrm{kpc})$ \\
\hline A & $100.7^{\mathrm{R}}$ & 4.3 & 7.5 & {$[25]$} & 4.2 & 8.6 \\
B & $102.4^{\mathrm{R}}$ & 4.3 & 8.7 & {$[24]$} & 4.2 & 8.5 \\
C & $86^{\mathrm{H}}$ & 4.6 & 9.6 & {$[4]$} & $\ldots$ & $\ldots$ \\
D & $96.6^{\mathrm{R}}$ & 4.4 & 5.8 & {$[23]$} & 4.3 & 9.0 \\
D' & $41.9 / 92.8^{\mathrm{R}}$ & 6.0 & $\ldots$ & {$[23]$} & $6.1 / 4.4$ & $11.9 / 9.2$ \\
E & $\ldots$ & $\ldots$ & $\ldots$ & $\ldots$ & 4.3 & 9.0 \\
F & $86^{\mathrm{H}}$ & 4.6 & 9.6 & {$[4]$} & $\ldots$ & $\ldots$ \\
G & $86^{\mathrm{H}}$ & 4.6 & 9.6 & {$[4]$} & $\ldots$ & $\ldots$ \\
\hline
\end{tabular}

${ }^{a}$ The superscripts $\mathrm{R}$ and $\mathrm{H}$ indicates $\mathrm{RRL}$ and $\mathrm{HI}$ absorption velocities respectively. ${ }^{\mathrm{b}}$ Literature values of Galactocentric radius (R) and distance $(\mathrm{d}) .{ }^{\mathrm{c}} \mathrm{Re}$-calculated Galactocentric radius $(\mathrm{R})$ and distance $(\mathrm{d})$ using the [21] rotation curve. 
the spectra are so similar. We deduce that the SNR candidate is at the same distance of $9.0 \pm 0.3 \mathrm{kpc}$.

\section{Discussion}

We derive a distance to the SNR G18.9-1.1 of $2.1 \pm 0.4 \mathrm{kpc}$, because of the observed absorption up to but not beyond the velocity of $23 \mathrm{~km} \cdot \mathrm{s}^{-1}$. In some cases, lack of absorption beyond a particular velocity could be interpreted as lack of absorbing $\mathrm{HI}$ at higher velocities. However, in this case, the $\mathrm{HI}$ emission at higher velocities is comparable to the emission at $\sim 23 \mathrm{~km} \cdot \mathrm{s}^{-1}$. Thus, if the SNR was located beyond $\sim 23 \mathrm{~km} \cdot \mathrm{s}^{-1}$, it would show HI absorption.

Furthermore, we examined the absorption spectra of HII regions 19.050 0.593 and $18.881-0.493$ which are $\sim 30^{\prime}$ and $\sim 35^{\prime}$ from the SNR center, respectively. The continuum brightness temperature of the HII region $19.050-0.593$ has maximum of $47.9 \mathrm{~K}$ which is comparable to the maximum brightness temperature of the SNR at $34.9 \mathrm{~K}$. The RRL velocity of the HII region is $68.2 \pm 1.3$ $\mathrm{km} \cdot \mathrm{s}^{-1}$ [24]. From the HI channel maps, it is seen that strong absorption features are present at $53.78 \mathrm{~km} \cdot \mathrm{s}^{-1}$ and weaker absorption features at $68.62 \mathrm{~km} \cdot \mathrm{s}^{-1}$, consistent with the RRL velocity. Using the Galactic rotation curve, the HII region is at a distance of $4.3 \pm 0.2 \mathrm{kpc}$. The HII region $18.881-0.493$ has a RRL velocity of $65.5 \pm 0.8 \mathrm{~km} \cdot \mathrm{s}^{-1}$. Strong absorption for $18.881-0.493$ is seen up to $70.26 \mathrm{~km} \cdot \mathrm{s}^{-1}$ and the $\mathrm{HI}$ absorption velocity is consistent with the RRL velocity within the radial velocity error of $5.3 \mathrm{~km} \cdot \mathrm{s}^{-1}$ [4]. $18.881-0.493$ is at a distance of $4.2 \pm 0.2$ $\mathrm{kpc}$. Thus, there is good evidence for absorbing $\mathrm{HI}$ at velocities higher than the observed absorption in SNR G18.9-1.1. We conclude the SNR is at the kinematic distance of $2.1-0.4 \mathrm{kpc}$, and consistent with [10] and [22].

G18.9-1.1 was observed in X-rays with the ROSAT PSPC [26] and ASCA [27]. We adopt a plasma electron temperature of $1.12 \mathrm{keV}$ from [27] and use the average of the GIS lower emission measure (EM) norm limit and the ROSAT upper EM norm limit together with our distance to estimate a value for EM of $2.6 \times$ $10^{57} \mathrm{~cm}^{-3}$. With the measured radius, electron temperature and emission measure, we apply the SNR models given in [28].

These models yield the age, explosion energy and ISM density that result in a SNR with the observed parameters. Table 2 lists the results of our modelling of G18.9-1.1. The SNR age is intermediate, between 2500 and $5000 \mathrm{yr}$, so that electron heating by ion collisions not yet complete. Assuming $T_{\mathrm{e}}=T_{\mathrm{i}}$ (i.e. $T_{\mathrm{e}}$ too large) gives a too-large age and too-low explosion energy, in effect making the SNR older and weaker than its actual age in an attempt to match the observed electron temperature. We see that the realistic models, with non-zero ejecta mass, yield ages which depend on assumed ejecta mass. The energy and ISM density also depends on ejecta mass but not as strongly. The SNR models also predict the temperature and emission measure of the reverse-shocked ejecta. When better X-ray observations are available, observation of the reverseshocked ejecta can distinguish between models with different ejecta mass and 
Table 2. SNR models for G18.9-1.1.

\begin{tabular}{ccccc}
\hline Model & $\begin{array}{c}\mathrm{M}_{\text {ejecta }}{ }^{\mathrm{a}} \\
\left(\mathrm{M}_{\odot}\right)\end{array}$ & $\begin{array}{c}\text { Age } \\
(\mathrm{yr})\end{array}$ & $\begin{array}{c}\mathrm{E}_{51} \\
\left(10^{51} \mathrm{erg}\right)\end{array}$ & $\begin{array}{c}\mathrm{n}_{0} \\
\left(\mathrm{~cm}^{-3}\right)\end{array}$ \\
\hline Sedov/ $T_{\mathrm{e}}=T_{\mathrm{i}}^{\mathrm{b}}$ & 0 & 4000 & 0.13 & $6.8 \times 10^{-2}$ \\
Sedov & 0 & 3300 & 0.27 & $9.5 \times 10^{-2}$ \\
$\mathrm{WL}^{\mathrm{c}}$ & 0 & 2600 & 0.45 & $2.6 \times 10^{-2}$ \\
Standard $/ s=0, n=7^{\mathrm{d}}$ & 1.4 & 3700 & 0.28 & $9.2 \times 10^{-2}$ \\
Standard $/ s=0, n=7^{\mathrm{d}}$ & 5.0 & 4700 & 0.26 & $8.0 \times 10^{-2}$ \\
\hline
\end{tabular}

${ }^{a}$ Ejecta mass, $\mathrm{M}_{\text {ejecta }}$ is an input value. ${ }^{\mathrm{b}}$ Assumes $T_{\mathrm{e}}=T_{\mathrm{i}}$. All other models include electron heating by Coulomb collisions. 'WL: cloudy ISM model of [29] with $\mathrm{C} / \tau=4$. ${ }^{\mathrm{d}}$ Standard model of [28], based on unified SNR evolution of [30]. $\mathrm{s}=0$ is for uniform ISM, $\mathrm{n}=7$ is for ejecta density profile $\propto r^{-7}$.

ejecta density profiles (the value of $\mathrm{n}$ ).

For SNR candidate G28.6+0.0, we measure its diameter as 2.9 arcmin using the VGPS radio continuum image. Our derived distance then gives its radius as $\mathrm{R}=3.8 \mathrm{pc}$. Because there is no reported $\mathrm{X}$-ray emission, we cannot apply models which use the X-ray emission to determine explosion energy $E_{0}$, ISM density $n$, and age [28]. Thus, we apply a basic Sedov model with the assumption of $E_{0}=$ $0.5 \times 10^{51} \mathrm{erg}$. This is the mean value found for Galactic SNRs and for LMC SNRs ([7] [31]). Then we take trial values of ISM density $n=0.1,1,10$ and 100 $\mathrm{cm}^{-3}$ to obtain ages of $180,580,1800$ and $5800 \mathrm{yr}$, respectively. In short, the small observed radius implies a small age unless the density is high.

\section{Summary}

In this work, we analyze HI spectra of SNR G18.9-1.1 and SNR candidate G28.6+0.0. ${ }^{13} \mathrm{CO}$ emission spectra and HI spectra of HII regions in the area are compared to the spectra of our objects to verify their kinematic distances. The distance of G18.9-1.1 is $2.1 \pm 0.4 \mathrm{kpc}$ and of G28.6+0.0 is $9.0 \pm 0.3 \mathrm{kpc}$.

Our derived radius of G18.9-1.1, with published X-ray shocked-ISM temperature and emission measures, allow application of SNR models. The models show that G18.9-1.1 has slightly low explosion energy of $\sim 3 \times 10^{50} \mathrm{erg}$ and is in a low-density region of the ISM, $\sim 0.1 \mathrm{~cm}^{-3}$. If the ejecta mass is $1.4 \mathrm{M}_{\odot}$, typical of Type Ia SNe, its age is $\sim 3700$. If the ejecta mass is $5 \mathrm{M}_{\odot}$, typical of core-collapse $\mathrm{SN}$, its age is $\sim 4700 \mathrm{yr}$. We apply a basic Sedov model to SNR candidate G18.9-1.1. If the ISM density is $1 \mathrm{~cm}^{-3}$ its Sedov age is $\sim 600 \mathrm{yr}$, but if the ISM density is as high as $100 \mathrm{~cm}^{-3}$ its age is significantly larger, $\sim 6000 \mathrm{yr}$.

\section{Acknowledgements}

This work was supported by a grant to DL from the Natural Sciences and Engineering Research Council of Canada.

\section{Conflicts of Interest}

The authors declare no conflicts of interest regarding the publication of this paper. 


\section{References}

[1] Green, D.A. (2019) VizieR Online Data Catalog: A Catalogue of Galactic Supernova Remnants (Green 2019). VizieR On-Line Data Catalog: VII/284. Cavendish Laboratory, Cambridge.

[2] Ranasinghe, S. and Leahy, D.A. (2017) Distances to Supernova Remnants G31.9+0.0 and G54.4-0.3 Associated with Molecular Clouds. The Astrophysical Journal, 843, 119-126. https://doi.org/10.3847/1538-4357/aa7894

[3] Ranasinghe, S. and Leahy, D.A. (2018) Revised Distances to 21 Supernova Remnants. The Astronomical Journal, 155, 204-215. https://doi.org/10.3847/1538-3881/aab9be

[4] Ranasinghe, S. and Leahy, D.A. (2018) Distances to Supernova Remnants G20.4+0.1, G24.7-0.6, and G28.6-0.1 and New Molecular Cloud Associations. Monthly Notices of the Royal Astronomical Society, 477, 2243-2250. https://doi.org/10.1093/mnras/sty817

[5] Ranasinghe, S., Leahy, D.A. and Tian, W.W. (2018) New Distances to Four Supernova Remnants. Open Physics Journal, 4, 1-13. https://doi.org/10.2174/1874843001804010001

[6] Tian, W.W., et al. (2019) The Kinematic Distances of SNR G16.7+0.1 and G15.9+0.2 by Analyzing HI Absorption Spectra. Publications of the Astronomical Society of the Pacific, 131, 114301-114312. https://doi.org/10.1088/1538-3873/ab35f4

[7] Leahy, D.A. and Ranasinghe, S. (2018) Evolutionary Models for 15 Galactic Supernova Remnants with New Distances. The Astrophysical Journal, 866, 9. https://doi.org/10.3847/1538-4357/aade48

[8] Fürst, E., Reich, W., Reich, P., Sofue, Y. and Handa, T. (1985) A New Non-Thermal Galactic Radio Source with a Possible Binary System. Nature, 314, 720-721.

https://doi.org/10.1038/314720a0

[9] Odegard, N. (1986) $57.5 \mathrm{MHz}$ Observations of Extended Nonthermal Sources in the Galactic Plane. The Astronomical Journal, 92, 1372-1380. https://doi.org/10.1086/114270

[10] Fürst, E., et al. (1989) A Study of the Composite Supernova Remnant G 18.95-1.1. Astronomy and Astrophysics, 209, 361-368.

[11] Sun, X.H., et al. (2011) A Sino-German $\lambda 6 \mathrm{~cm}$ Polarization Survey of the Galactic plane. VII. Small Supernova Remnants. Astronomy and Astrophysics, 536, A83. https://doi.org/10.1051/0004-6361/201117693

[12] Anderson, L.D., et al. (2017) Galactic Supernova Remnant Candidates Discovered by THOR. Astronomy and Astrophysics, 605, A58. https://doi.org/10.1051/0004-6361/201731019

[13] Helfand, D.J., Velusamy, T., Becker, R.H. and Lockman, F.J. (1989) The Prevalence of Supernova Remnants among Unidentified Galactic Radio Sources. The Astrophysical Journal, 341, 151-162. https://doi.org/10.1086/167479

[14] Bamba, A., Ueno, M., Koyama, K. and Yamauchi, S. (2001) A Diffuse X-Ray Source, AX J1843.8-0352: Association with the Radio Complex G28.6-0.1 and Identification of a New Supernova Remnant. Publications of the Astronomical Society of Japan, 53, L21-L24. https://doi.org/10.1093/pasj/53.4.L21

[15] Ferrand, G. and Safi-Harb, S. (2012) A Census of High-Energy Observations of Galactic Supernova Remnants. Advances in Space Research, 49, 1313-1319. https://doi.org/10.1016/j.asr.2012.02.004 
[16] Stil, J.M., et al. (2006) The VLA Galactic Plane Survey. The Astronomical Journal, 132, 1158-1176. https://doi.org/10.1086/505940

[17] Jackson, J.M., et al. (2006) The Boston University-Five College Radio Astronomy Observatory Galactic Ring Survey. The Astrophysical Journal Supplement Series, 163, 145-159. https://doi.org/10.1086/500091

[18] Leahy, D. and Tian, W. (2010) Distances to Supernova Remnants from H I Absorption Spectra. The Dynamic Interstellar Medium: A Celebration of the Canadian Galactic Plane Survey, 438, 365.

[19] Beuther, H., et al. (2016) The HI/OH/Recombination Line Survey of the Inner Milky Way (THOR). Survey Overview and Data Release 1. Astronomy and Astrophysics, 595, A32. https://doi.org/10.1051/0004-6361/201629143

[20] Persic, M., Salucci, P. and Stel, F. (1996) The Universal Rotation Curve of Spiral Galaxies-I. The Dark Matter Connection. Monthly Notices of the Royal Astronomical Society, 281, 27-47. https://doi.org/10.1093/mnras/278.1.27

[21] Reid, M.J., et al. (2014) Trigonometric Parallaxes of High Mass Star Forming Regions: The Structure and Kinematics of the Milky Way. The Astrophysical Journal, 783, 130-143. https://doi.org/10.1088/0004-637X/783/2/130

[22] Shan, S.S., et al. (2018) Distances of Galactic Supernova Remnants Using Red Clump Stars. The Astrophysical Journal Supplement Series, 238, 35-46. https://doi.org/10.3847/1538-4365/aae07a

[23] Anderson, L.D., et al. (2015) Finding Distant Galactic HII Regions. The Astrophysical Journal Supplement Series, 221, 26-36. https://doi.org/10.1088/0067-0049/221/2/26

[24] Lockman, F.J. (1989) A Survey of Radio H II Regions in the Northern Sky. The Astrophysical Journal Supplement Series, 71, 469-479. https://doi.org/10.1086/191383

[25] Anderson, L.D., Bania, T.M., Balser, D.S. and Rood, R.T. (2011) The Green Bank Telescope H II Region Discovery Survey. II. The Source Catalog. The Astrophysical Journal Supplement Series, 194, 32-51. https://doi.org/10.1088/0067-0049/194/2/32

[26] Fürst, E., Reich, W. and Aschenbach, B. (1997) New Radio and Soft X-Ray Observations of the Supernova Remnant G 18.95-1.1. Astronomy and Astrophysics, 319, 655-663.

[27] Harrus, I.M., Slane, P.O., Hughes, J.P. and Plucinsky, P.P. (2004) An X-Ray Study of the Supernova Remnant G18.95-1.1. The Astrophysical Journal, 603, 152-158. https://doi.org/10.1086/381355

[28] Leahy, D., Wang, Y., Lawton, B., Ranasinghe, S. and Filipović, M. (2019) Emission Measures and Emission-Measure-Weighted Temperatures of Shocked Interstellar Medium and Ejecta in Supernova Remnants. The Astronomical Journal, 158, 149-166. https://doi.org/10.3847/1538-3881/ab3d2c

[29] White, R.L. and Long, K.S. (1991) Supernova Remnant Evolution in an Interstellar Medium with Evaporating Clouds. The Astrophysical Journal, 373, 543-555. https://doi.org/10.1086/170073

[30] Truelove, J.K. and McKee, C.F. (1999) Evolution of Nonradiative Supernova Remnants. The Astrophysical Journal Supplement Series, 120, 299-326. https://doi.org/10.1086/313176

[31] Leahy, D.A. (2017) Energetics and Birth Rates of Supernova Remnants in the Large Magellanic Cloud. The Astrophysical Journal, 837, 36-41.

https://doi.org/10.3847/1538-4357/aa60c1 